\title{
UNIFORM (PROJECTIVE) HYPERBOLICITY OR NO HYPERBOLICITY: A DICHOTOMY FOR GENERIC CONSERVATIVE MAPS
}

\author{
Jairo BOCHI, Marcelo VIANA ${ }^{1}$ \\ IMPA - Instituto de Matemática Pura e Aplicada, Est. D. Castorina 110, \\ 22460-320 Rio de Janeiro, Brazil
}

Received 25 September 2001

ABSTRACT. - We show that the Lyapunov exponents of volume preserving $C^{1}$ diffeomorphisms of a compact manifold are continuous at a given diffeomorphism if and only if the Oseledets splitting is either dominated or trivial. It follows that for a $C^{1}$-residual subset of volume preserving diffeomorphisms the Oseledets splitting is either dominated or trivial.

We obtain analogous results in the setting of symplectic diffeomorphisms, where the conclusion is actually stronger: dominated splitting is replaced by partial hyperbolicity. We also obtain versions of these results for continuous cocycles with values in some matrix groups.

In the text we give the precise statements of these results and the ideas of the proofs. The complete proofs will appear in [4].

(C) 2002 L'Association Publications de l'Institut Henri Poincaré. Published by Elsevier B.V. All rights reserved

AMS classification: 37C40; 37A35; 7A20

RÉSUMÉ. - Nous montrons que les exposants de Lyapunov des difféomorphismes de classe $C^{1}$ qui préservent le volume dans une varieté compacte sont continus à un difféomorphisme donné si et seulement si sa décomposition de Oseledets est dominée ou bien triviale. Il s'en suit que pour un sous-ensemble $C^{1}$-résiduel des difféomorphismes qui préservent le volume, la décomposition de Oseledets est soit dominée soit triviale.

Nous obtenons des résultats analogues dans le cadre des difféomorphismes symplectiques où, en fait, les conclusions sont plus fortes : on remplace décomposition dominée par hyperbolicité partielle. De même, nous obtenons des versions de ces résultats pour des cocyles continus à valeurs dans plusieurs groupes de matrices.

Dans le texte en anglais nous donnons les énoncés précis de ces résultats, et des idées des preuves. Les démonstrations complètes apparaitront dans [4].

(C) 2002 L'Association Publications de l'Institut Henri Poincaré. Published by Elsevier B.V. All rights reserved

E-mail addresses: bochi@impa.br (J. Bochi),viana@impa.br (M. Viana).

${ }^{1}$ Partially supported by Faperj, CNPq-001/2000, and Pronex-Dynamical Systems. 


\section{Introduction}

The results presented in this paper exploit a connection between the following a priori loosely related problems: How do Lyapunov exponents of conservative (symplectic or volume preserving) diffeomorphisms depend on the underlying dynamics? How typical is it for Lyapunov exponents to vanish?

We prove that Lyapunov exponents can be simultaneously continuous at a given $C^{1}$ diffeomorphism only if the corresponding Oseledets splitting is dominated or else trivial, almost everywhere.

Trivial splitting means that all Lyapunov exponents are equal to zero. Domination is a property of uniform hyperbolicity on the projective bundle, whose precise definition will be recalled in a while.

As a consequence one gets a surprising dichotomy for a residual (dense $G_{\delta}$ ) subset of volume preserving $C^{1}$ diffeomorphisms on any compact manifold: the Oseledets splitting is either dominated or trivial, almost everywhere.

Analogous results hold for symplectic diffeomorphisms, where the conclusion is even stronger: domination is replaced by partial hyperbolicity.

Moreover, there are versions of these statements for continuous cocycles with values in various matrix Lie groups.

In the sequel we give the precise statements, and ideas of the proofs. For that, we begin by explaining the meaning and significance of the domination property. Complete proofs of these statements will appear in [4].

\section{Lyapunov exponents}

1. Let $f: M \rightarrow M$ be a $C^{1}$ diffeomorphism preserving the volume $\mu$ of a compact Riemannian manifold $M$. Oseledets theorem [11] states that, for $\mu$-almost every $x \in M$, there exists a splitting

$$
T_{x} M=E_{x}^{1} \oplus \cdots \oplus E_{x}^{k}, \quad k=k(x) \in \mathbb{N},
$$

of the tangent space, and there exist real numbers $\lambda_{1}(x)>\cdots>\lambda_{k}(x)$ such that

$$
\left\|D f^{n}(x) v_{j}\right\| \approx \mathrm{e}^{n \lambda_{j}(x)}\left\|v_{j}\right\|
$$

for all $v_{j} \in E_{x}^{j}$ and $1 \leqslant j \leqslant k$, if $|n|$ is large enough. More precisely,

$$
\lim _{n \rightarrow \pm \infty} \frac{1}{n} \log \left\|D f^{n}(x) v_{j}\right\|=\lambda_{j}(x) \quad \text { for all } v_{j} \in E_{x}^{j} \backslash\{0\} .
$$

The Oseledets subspaces $E_{x}^{j}$ and the Lyapunov exponents $\lambda_{j}(x)$ depend measurably on $x$, and they are invariant under the dynamics:

$$
\lambda_{j}(f(x))=\lambda_{j}(x) \quad \text { and } \quad E_{f(x)}^{j}=D f(x) \cdot E_{x}^{j}, \quad \text { with } k(f(x))=k(x) .
$$


2. In particular, one can always find $m(x) \in \mathbb{N}$ such that

$$
\left\|D f^{n}(x) v_{i}\right\| \geqslant 2\left\|D f^{n}(x) v_{j}\right\| \text { for all } n \geqslant m(x)
$$

and all norm 1 vectors $v_{i} \in E_{x}^{i}$ and $v_{j} \in E_{x}^{j}$ with $1 \leqslant i<j \leqslant k$. That is, iterates of $D f$ are eventually more expanding along $E_{x}^{i}$ than along $E_{x}^{j}$, for any $i<j$.

Let us stress, however, that this is a purely asymptotic statement. The limit in (1) is usually not uniform on $x$. Correspondingly, the value of $m(x)$ in (2) does depend on the point, in a complicated fashion. Most important, in general it is not even possible to choose $m(\cdot)$ bounded over each orbit $o(x)=\left\{f^{\ell}(x): \ell \in \mathbb{Z}\right\}$.

\section{Domination property}

3. We say that the Oseledets splitting is dominated at $x \in M$ if $m(\cdot) \in \mathbb{N}$ as in (2) may be chosen uniform over the whole orbit of $x$. That is, domination at $x$ means that there is $m \in \mathbb{N}$ such that for every iterate $y=f^{\ell}(x), \ell \in \mathbb{Z}$, we have

$$
\left\|D f^{n}(y) v_{i}\right\| \geqslant 2\left\|D f^{n}(y) v_{j}\right\| \text { for all } n \geqslant m
$$

and all norm 1 vectors $v_{i} \in E_{y}^{i}, v_{j} \in E_{y}^{j}$, and $1 \leqslant i<j \leqslant k$.

More generally, we say that the Oseledets splitting is dominated over an invariant set $\Gamma$ if there exists $m \in \mathbb{N}$ such that (3) is satisfied for every $y \in \Gamma, n \geqslant m, 1 \leqslant i<j \leqslant k(y)$, and $v_{i}, v_{j}$ as before. The definition in the previous paragraph corresponds to the case $\Gamma=o(x)$, of course.

4. Geometrically, domination is tantamount to uniform hyperbolicity of the dynamics induced on the projective bundle, i.e. the bundle of directions in the tangent space. We explain this with the aid of Fig. 1.

From the relation (3) one easily deduces that

$$
\left\|D f^{n}(y) v_{i}\right\| \geqslant c 2^{n / m}\left\|D f^{n}(y) v_{j}\right\| \text { for every } n \geqslant 1
$$

and for all $y \in o(x), 1 \leqslant i<j \leqslant k$, and norm 1 vectors $v_{i} \in E_{y}^{i}$ and $v_{j} \in E_{y}^{j}$, where the constant $c>0$ depends only on $m$ and $f$. Then, given any $y \in o(x)$ and non-zero

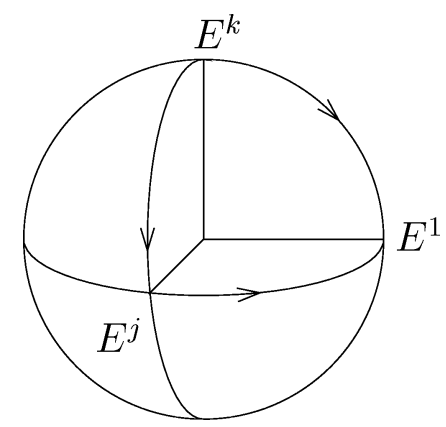

Fig. 1. Uniform projective hyperbolicity. 
$v \in T_{y} M$, let $v=v_{1}+\cdots+v_{k}$ be the splitting of $v$ along Oseledets subspaces, and $1 \leqslant p \leqslant q \leqslant k$ be, respectively, smallest and largest such that $v_{p} \neq 0 \neq v_{q}$.

From (4) one gets that, as $n$ increases to infinity the component $D f^{n}(y) v_{p}$ becomes much larger than any other $D f^{n}(y) v_{j}$. So, $D f^{n}(y) v$ approaches the direction of $E_{f^{n}(y)}^{p}$, exponentially fast. There is also a dual statement for large negative $n$, with $p$ replaced by $q$. Clearly, for most vectors $p=1$ and $q=k$. This means that $E^{1}$ is a global hyperbolic attractor and $E^{k}$ is a global hyperbolic repeller for the dynamics of $D f$ on the projective bundle, with the other $E^{j}$ 's in the role of saddles.

5. It is not difficult to find open sets of maps whose Oseledets splitting is dominated, e.g., any small neighborhood of a linear torus automorphism whose eigenvalues have multiplicity 1 and different norms. Notwithstanding, domination is really a very rigid property.

For one thing, if the number $k(y)$ and the dimensions $d_{j}(y)=\operatorname{dim} E_{y}^{j}$ of the Oseledets subspaces are constant on $\Gamma$, domination implies that the splitting is continuous on $\Gamma$, and even admits a continuous extension to the closure. In particular, the angles between the various Oseledets subspaces are bounded from zero, uniformly over the invariant set $\Gamma$. Actually, this last fact remains true even if the number or the dimensions of the subspaces are variable. That is because we can always partition $\Gamma$ into a finite number of invariant subsets corresponding to fixed values of $k$ and $d_{1}, \ldots, d_{k}$.

Due to this rigidity, it is often possible to exclude a priori the existence of dominated splitting, using topological arguments. One such situation will appear near the end of Paragraph 8, another in Paragraph 18.

\section{A global picture for generic conservative maps}

6. $\operatorname{Diff}_{\mu}^{1}(M)$ denotes the space of volume preserving $C^{1}$ diffeomorphisms on $M$, endowed with the $C^{1}$ topology. Our first main result is

THEOREM 1. - For any compact manifold $M$, there exists a residual subset $\mathcal{R}$ of $\operatorname{Diff}_{\mu}^{1}(M)$ such that, for every $f \in \mathcal{R}$, the Oseledets splitting is dominated or else trivial, at almost every point.

Later we shall explain how Theorem 1 may be derived from a statement about continuity of Lyapunov exponents. Right now let us discuss the conclusion of the theorem in more detail.

First, let us consider $f \in \mathcal{R}$ to be ergodic. Then the number and dimensions of the Oseledets subspaces are constant $\mu$-almost everywhere. The theorem says that,

- either all Lyapunov exponents vanish $\mu$-almost everywhere,

- or the Oseledets splitting is dominated, also $\mu$-almost everywhere.

In the latter case, the splitting extends continuously to the whole manifold: the system is projectively uniformly hyperbolic on $M$.

7. In general, let $\mathcal{O}\left(d_{1}, \ldots, d_{k}\right)$ be the set of points for which the Oseledets splitting exists and involves $k$ subspaces, with dimensions $d_{1}, \ldots, d_{k}$, respectively. In particular $\mathcal{O}(d)$ denotes the set of points whose Oseledets splitting is trivial, that is, whose Lyapunov exponents are all zero. 
For $k \geqslant 2$, let $\mathcal{O}_{\text {dom }}\left(d_{1}, \ldots, d_{k}\right) \subset \mathcal{O}\left(d_{1}, \ldots, d_{k}\right)$ be the subset of points where the Oseledets splitting is dominated. Theorem 1 says that

$$
\mu\left(\mathcal{O}\left(d_{1}, \ldots, d_{k}\right) \backslash \mathcal{O}_{\mathrm{dom}}\left(d_{1}, \ldots, d_{k}\right)\right)=0
$$

for all $k \geqslant 2$ and any choice of $d_{1}, \ldots, d_{k}$. Therefore,

$$
M=\mathcal{O}(d) \cup \bigcup_{k \geqslant 2} \bigcup_{d_{1}, \ldots, d_{k}} \mathcal{O}_{\mathrm{dom}}\left(d_{1}, \ldots, d_{k}\right)
$$

up to a zero volume set.

$\mathcal{O}_{\text {dom }}\left(d_{1}, \ldots, d_{k}\right)$ may be written as an increasing union

$$
\mathcal{O}_{\text {dom }}\left(d_{1}, \ldots, d_{k}\right)=\bigcup_{m=1}^{\infty} \mathcal{O}_{m}\left(d_{1}, \ldots, d_{k}\right)
$$

where each $\mathcal{O}_{m}\left(d_{1}, \ldots, d_{k}\right)$ corresponds to fixing the choice of $m$ in (3). The Oseledets splitting is continuous on every $\mathcal{O}_{m}\left(d_{1}, \ldots, d_{k}\right)$, and extends continuously to the closure. In general, these extensions need not coincide on the intersections of the closures.

8. As the reader may easily check, for area preserving diffeomorphisms on surfaces, domination is equivalent to uniform hyperbolicity (in the usual sense, no projectivisation). On the other hand, for a residual subset of $C^{1}$ diffeomorphisms on any manifold, ${ }^{2}$ hyperbolic sets have a sort of automatic ergodicity: either they have zero volume or they coincide with the whole ambient space.

Because of this, in dimension 2 the conclusion of Theorem 1 is simpler:

THEOREM 2 ([3], partially based on [10]). - For a residual subset of area preserving $C^{1}$ diffeomorphisms on any compact surface, both Lyapunov exponents are zero at almost every point or else the diffeomorphism is Anosov.

It would be nice to know whether this simpler picture remains true in arbitrary dimension, without assuming ergodicity:

Problem 1. - Is there a residual set $\mathcal{R}_{1} \subset \mathcal{R}$ for which, in the context of (5)-(6), either $\mathcal{O}(d)$ or some $\mathcal{O}_{m}\left(d_{1}, \ldots, d_{k}\right)$ has full volume in $M$ ?

Corollary 1 below gives a partial answer, for symplectic maps.

Also, existence of Anosov diffeomorphisms imposes strong topological restrictions on the manifold. In particular, the second alternative in Theorem 2 is possible only if $M=\mathbb{T}^{2}$.

Problem 2. - Which manifolds support diffeomorphisms having a dominated splitting defined on the whole ambient space?

\footnotetext{
${ }^{2}$ This subset includes all $C^{2}$ diffeomorphisms [6]. A corresponding statement for domination is false if $d>3$ : there exist $C^{\infty}$ diffeomorphisms exhibiting invariant sets supporting a dominated splitting and whose volume is neither zero nor full.
} 


\section{Continuity of Lyapunov exponents}

9. Let $T_{x} M=E_{x}^{1} \oplus \cdots \oplus E_{x}^{k}$ be the Oseledets splitting of $f \in \operatorname{Diff}_{\mu}^{1}(M)$ at some point $x \in M$. Let

$$
\hat{\lambda}_{1}(x) \geqslant \hat{\lambda}_{2}(x) \geqslant \cdots \geqslant \hat{\lambda}_{d}(x), \quad d=\operatorname{dim} M,
$$

be the Lyapunov exponents of $f$ at $x$, counted with multiplicity: each $\hat{\lambda}_{j}(x)$ appears exactly $d_{j}(x)=\operatorname{dim} E_{x}^{j}$ times. Formally,

$$
\hat{\lambda}_{i}(x)=\lambda_{j}(x) \quad \text { if } 1 \leqslant i-\left[d_{1}(x)+\cdots+d_{j-1}(x)\right] \leqslant d_{j}(x) .
$$

This defines measurable functions $\hat{\lambda}_{i}, 1 \leqslant i \leqslant d$, over a full measure subset of the manifold $M$.

Now we consider the average Lyapunov exponents of a diffeomorphism $f$, given by $\hat{\lambda}_{i}(f)=\int \hat{\lambda}_{i}(x) \mathrm{d} \mu(x)$, for $1 \leqslant i \leqslant d$. This defines functions

$$
\hat{\lambda}_{i}: \operatorname{Diff}_{\mu}^{1}(M) \rightarrow \mathbb{R}, \quad 1 \leqslant i \leqslant d .
$$

Note that $\hat{\lambda}_{1}(x)+\cdots+\hat{\lambda}_{d}(x)=0$, because $f$ preserves volume. Hence,

$$
\hat{\lambda}_{1}(f)+\cdots+\hat{\lambda}_{d}(f)=0 \quad \text { for every } f \in \operatorname{Diff}_{\mu}^{1}(M) .
$$

10. Another main result, from which we shall deduce Theorem 1 , is

THEOREM 3. - Suppose $f_{0} \in \operatorname{Diff}_{\mu}^{1}(M)$ is a continuity point for the map

$$
\operatorname{Diff}_{\mu}^{1}(M) \ni f \mapsto\left(\hat{\lambda}_{1}(f), \ldots, \hat{\lambda}_{d}(f)\right) \in \mathbb{R}^{d} .
$$

Then, at almost every point, the Oseledets splitting of $f_{0}$ is either dominated or trivial.

The converse is true, and much easier. See comments in Paragraph 17.

\section{Symplectic diffeomorphisms}

11. The previous results extend to the symplectic case. Let $M$ be a compact manifold of dimension $d=2 l$, endowed with a symplectic form $\omega$. Let $\operatorname{Symp}_{\omega}^{1}(M)$ be the space of $\omega$-preserving $C^{1}$ diffeomorphisms on $M$. This is a closed subset of the space $\operatorname{Diff}_{\mu}^{1}(M)$ of $C^{1}$ diffeomorphisms that preserve the volume measure $\mu$ induced by the volume form $\omega^{l}=\omega \wedge \cdots \wedge \omega$.

THEOREM 4. - Theorems 1 and 3 remain true when one replaces $\operatorname{Diff}_{\mu}^{1}(M)$ by $\operatorname{Symp}_{\omega}^{1}(M)$. Actually, in the symplectic context the conclusion is stronger: instead of domination one gets partial hyperbolicity.

The present notion of partial hyperbolicity is also stronger than usual, in that the central bundle exhibits only zero Lyapunov exponents, and the dimensions of the stable bundle and the unstable bundle are equal: 
12. The Oseledets splitting of a symplectic diffeomorphism has a symmetric structure:

$$
T_{x} M=E_{x}^{s} \oplus \cdots \oplus E_{x}^{1} \oplus\left[E_{x}^{0} \oplus\right] E_{x}^{-1} \oplus \cdots \oplus E_{x}^{-s}
$$

with $\operatorname{dim} E_{x}^{i}=\operatorname{dim} E_{x}^{-i}$ for $1 \leqslant i \leqslant s$ (the dimension of $E_{x}^{0}$ may be zero) and Lyapunov exponents

$$
\lambda_{s}(x)>\cdots>\lambda_{1}(x)>\left[\lambda_{0}(x)=\right] 0>\lambda_{-1}(x)>\cdots>\lambda_{-s}(x)
$$

satisfying $\lambda_{i}(x)+\lambda_{-i}(x)=0$ for $1 \leqslant i \leqslant s$. Let

$$
E_{x}^{+}=E_{x}^{s} \oplus \cdots \oplus E_{x}^{1} \quad \text { and } \quad E_{x}^{-}=E_{x}^{-1} \oplus \cdots \oplus E_{x}^{-s} .
$$

We say that the Oseledets splitting is partially hyperbolic at $x$ if it is dominated at $x$ and $D f$ is uniformly expanding along $E^{+}$and uniformly contracting along $E^{-}$:

$$
\left\|D f^{-m} \mid E_{y}^{+}\right\| \leqslant \frac{1}{2} \quad \text { and } \quad\left\|D f^{m} \mid E_{y}^{-}\right\| \leqslant \frac{1}{2}
$$

for any iterate $y=f^{\ell}(x), \ell \in \mathbb{Z}$, where $m \in \mathbb{N}$ is uniform over the orbit of $x$.

Proposition 1.-Let $f$ be a symplectic diffeomorphism. If the Oseledets splitting of $f$ is dominated at a point $x$ then it is partially hyperbolic at $x$.

This fact was first observed by Mañé [9]. A proof is given in [1], for $\operatorname{dim} M=4$, and in [4], for the general case.

13. Theorem 4 has the following interesting consequence, that extends conclusions in Paragraph 8.

COROLLARY 1. - For a residual subset of $\operatorname{Symp}_{\mu}^{1}(M)$, either the diffeomorphism is Anosov or almost every point exhibits some Lyapunov exponent equal to zero (with even multiplicity).

Indeed, for $f \in \mathcal{R}$, the set of points whose Lyapunov exponents are all non-zero is a countable union of hyperbolic sets: the union is over the value of $m$ in (8). Hence, restricting to some residual $\mathcal{R}_{1} \subset \mathcal{R}$, either that set has zero measure, or the whole manifold is hyperbolic for $f$. Recall also that $\operatorname{dim} E_{x}^{0}$ is always an even number, in the symplectic case.

\section{The semi-continuity argument}

14. We are going to deduce Theorem 1 from Theorem 3 . For each $1 \leqslant i \leqslant d$, let

$$
\Lambda_{i}(f)=\hat{\lambda}_{1}(f)+\cdots+\hat{\lambda}_{i}(f) .
$$

The relation (7) means that $\Lambda_{d}(f) \equiv 0$. Clearly, $f \mapsto\left(\hat{\lambda}_{1}(f), \ldots, \hat{\lambda}_{d}(f)\right)$ is continuous at $f_{0}$ if and only if $f \mapsto\left(\Lambda_{1}(f), \ldots, \Lambda_{d-1}(f)\right)$ is continuous at $f_{0}$. 
Proposition 2. - Every $f \mapsto \Lambda_{i}(f), 1 \leqslant i \leqslant d-1$, is upper semi-continuous, both on $\operatorname{Diff}_{\mu}^{1}(M)$ and on $\operatorname{Symp}_{\omega}^{1}(M)$.

This proposition is proved as follows. For the largest Lyapunov exponent $\Lambda_{1}=\hat{\lambda}_{1}$ one uses the relation

$$
\hat{\lambda}_{1}(f)=\inf _{n \geqslant 1} \frac{1}{n} \int \log \left\|D f^{n}\right\| \mathrm{d} \mu .
$$

The infimum of continuous functions being upper semi-continuous, the conclusion follows.

For $i \geqslant 2$, one considers the vector bundle $V^{i}$ over $M$ whose fiber $V_{x}^{i}$ is the space of $i$-forms on $\left(T_{x} M\right)^{*}$. The derivative $D f$ induces a fibered map $D f^{\wedge i}: V^{i} \rightarrow V^{i}$, and the largest Lyapunov exponent of $D f^{\wedge i}$ is precisely $\Lambda_{i}(f)$ (see [8]). Thus we have the relation corresponding to (9) for $D f^{\wedge i}$ :

$$
\Lambda_{i}(f)=\inf _{n \geqslant 1} \frac{1}{n} \int \log \left\|\left(D f^{\wedge i}\right)^{n}\right\| \mathrm{d} \mu,
$$

and semi-continuity follows as before.

Remark 1. - A natural choice of a norm in (10) is such that the quantity $\left\|\left(D f^{\wedge i}(x)\right)^{n}\right\|$ is the supremum of the $i$-volume of $D f^{n}(x)(P)$ over $i$-parallelepipeds $P \subset T_{x} M$ of unit $i$-volume. Of course, any other norm would work as well.

Theorem 1 is now an immediate consequence of Theorem 3, Proposition 2, and the well-known fact that the set of continuity points of any semi-continuous function defined on a Baire space contains a residual subset.

\section{The perturbation strategy}

15. The proof of Theorem 3 is quite long. Here we only give a glimpse of the strategy to reduce Lyapunov exponents along finite pieces of orbits.

Recall that the Oseledets splitting is dominated at $x$ if condition (3) is satisfied. It is not hard to see that this condition can be reformulated as follows: There exists $m \in \mathbb{N}$ such that for every iterate $y=f^{\ell}(x), \ell \in \mathbb{Z}$,

$$
\left\|D f^{m}(y) v\right\| \geqslant 2\left\|D f^{m}(y) w\right\|
$$

for all norm 1 vectors $v \in E_{y}^{1} \oplus \cdots \oplus E_{y}^{i}$ and $w \in E_{y}^{i+1} \oplus \cdots \oplus E_{y}^{k}$, and all $1 \leqslant i \leqslant k-1$.

Now suppose the Oseledets splitting is not dominated, over a positive measure set of orbits: for some $i$ and for arbitrarily large $m$ there exist iterates $y$ for which (11) does not hold. The basic idea is to take advantage of this fact to, by a small perturbation of the map, cause a vector originally in $E_{y}^{1, i}=E_{y}^{1} \oplus \cdots \oplus E_{y}^{i}$ to move to $E_{f^{m}(y)}^{i+1, k}=$ $E_{f^{m}(y)}^{i+1} \oplus \cdots \oplus E_{f^{m}(y)}^{k}$, thus "blending" different expansion rates.

More precisely, given a perturbation size $\varepsilon>0$ one chooses $m$ sufficiently large with respect to $\varepsilon$. Then for $n \gg m$ one chooses a convenient $y=f^{\ell}(x)$, with $\ell \approx n / 2$, where domination fails. By composing $D f$ with small rotations near, at most, $m$ iterates of 


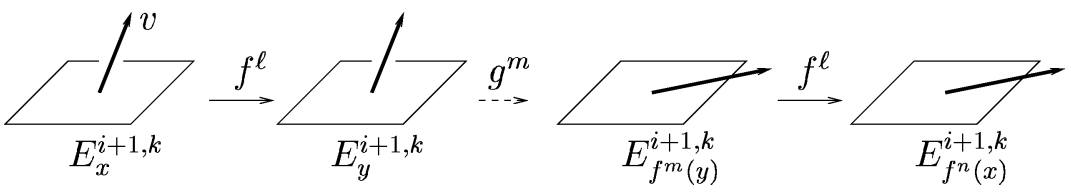

Fig. 2. Blending expansion rates.

$y$, one causes the orbit of some $v \in E^{1, i}$ to move to $E^{i+1, k}$. See Fig. 2. That is, one constructs a perturbed map $g$ preserving the orbit segment $\left\{x, \ldots, f^{n}(x)\right\}$ such that $D g^{n}(x) v \in E_{f^{n}(x)}^{i+1, k}$. As a result, it is possible to show that

$$
\left\|\left(D g^{\wedge j}(x)\right)^{n}\right\| \lesssim \exp \left[n\left(\Lambda_{j-1}+\frac{\hat{\lambda}_{j}+\hat{\lambda}_{j+1}}{2}\right)\right] \ll \exp \left(n \Lambda_{j}\right),
$$

where $j=\operatorname{dim} E^{1, i}$.

This local procedure is then repeated for several points $x$. Using (10) and a tower argument, one proves that $\Lambda_{j}(f)$ drops under such arbitrarily small perturbations, contradicting continuity.

\section{Linear cocycles over transformations}

16. The previous methods also extend to products of deterministic continuous matrices. In this setting one considers a measurable invertible transformation $f: M \rightarrow M$ on a compact space $M$, preserving a Borel probability measure $\mu$. The system should be aperiodic, meaning that the set of periodic points of $f$ should have zero $\mu$-measure. For simplicity, here we also take the system to be ergodic.

Then one considers the space of all continuous maps $A: M \rightarrow G$, where $G$ is a convenient matrix group. In all that follows we may take $G=S L(d, \mathbb{R}), G L(d, \mathbb{R})$, $S p(d), \ldots$, and we may also replace $\mathbb{R}$ by $\mathbb{C}$ as the field of coefficients. Associated to each $A$, one considers the cocycle

$$
F: M \times \mathbb{R}^{d} \rightarrow M \times \mathbb{R}^{d}, \quad F(x, v)=(f(x), A(x) v) .
$$

Note that $F^{n}(x, v)=\left(f^{n}(x), A^{n}(x) v\right)$, where

$$
A^{n}(x)=A\left(f^{n-1}(x)\right) \cdots A(f(x)) A(x) \quad \text { and } \quad A^{-n}(x)=\left[A^{n}\left(f^{-n}(x)\right)\right]^{-1},
$$

for $n \geqslant 1$. As before, Oseledets splittings $\{x\} \times \mathbb{R}^{d}=E_{x}^{1} \oplus \cdots \oplus E_{x}^{k}$ and Lyapunov exponents

$$
\lambda_{j}(x)=\lim _{n \rightarrow \pm \infty} \frac{1}{n} \log \left\|A^{n}(x) v_{j}\right\|, \quad v_{j} \in E_{x}^{j} \backslash\{0\},
$$

are well-defined $\mu$-almost everywhere.

17. We prove that for a residual subset of continuous maps $A: M \rightarrow G$, either the Oseledets splitting is dominated, and hence continuous, over the whole space $M$, 
or the Lyapunov exponents are all equal $(k=1)$ at $\mu$-almost every point. As before, this follows from proving that average Lyapunov exponents can be simultaneously continuous at some $A_{0}$ only if the Oseledets splitting of the cocycle associated to $A_{0}$ is either dominated or trivial.

The converse is also true: if the Oseledets splitting of $A_{0}$ is trivial or dominated then $A_{0}$ is a continuity point for all Lyapunov exponents. The proof has two main ingredients. The first one is the robustness of dominated (or trivial) splittings: every $C^{0}$ nearby map $A$ has an invariant splitting into subspaces with the same dimensions and uniformly close to the Oseledets subspaces of $A_{0}$. The second one is a semi-continuity argument within each of these subspaces, to show that its Lyapunov exponents are close to the corresponding exponents of $A_{0}$.

It is interesting to put this conclusion together with a theorem of Ruelle [12] stating that if the Oseledets splitting of $A_{0}$ is dominated, and the Oseledets subspaces all have dimension 1, then the Lyapunov exponents vary smoothly with the matrix map $A$ near $A_{0}$.

Oseledets subspaces of $C^{0}$ generic cocycles need not be 1-dimensional:

Example 1.- Let $f: M \rightarrow M$ have a fixed point $p$. Let $A_{0}: M \rightarrow S L(3, \mathbb{R})$ be constant, with one real and two complex conjugate eigenvalues. Assuming the norms of the eigenvalues are not all equal, the Oseledets splitting of $A_{0}$ is the (constant) splitting into eigenspaces, and it is dominated. Then every $A$ in a $C^{0}$ neighborhood $\mathcal{U}$ of $A_{0}$ admits a dominated splitting $F^{1} \oplus F^{2}$, with $\operatorname{dim} F_{x}^{i}=i$. In particular, the Lyapunov exponents of $A$ can not be all equal. So, for every $A \in \mathcal{U} \cap \mathcal{R}$ the Oseledets splitting must be dominated, and it must be a refinement of $F^{1} \oplus F^{2}$. Now, assuming $\mathcal{U}$ is sufficiently small, $F^{2}$ admits no continuous invariant proper subbundle. That is because $A(p)$ has complex eigenvalues along $F_{p}^{2}$. Hence, if $A \in \mathcal{U} \cap \mathcal{R}$ then its Oseledets splitting must coincide with $F^{1} \oplus F^{2}$ almost everywhere.

But [5] shows that, for appropriate choices of $(f, \mu)$, the majority of Hölder continuous maps $A \in \mathcal{U}$ (an open dense subset, whose complement has infinite codimension) do have all their Oseledets subspaces with dimension 1.

18. Here is one situation where simple topological reasons prevent the existence of dominated splittings, for a whole $C^{0}$ open set of cocycles:

Example 2. - Let $f: S^{1} \rightarrow S^{1}$ be a homeomorphism and $\alpha: S^{1} \rightarrow S^{1}$ be a continuous map with non-zero degree. Let $G=S L(2, \mathbb{R})$. Fix any $B \in G$ and define $A: S^{1} \rightarrow G$ by $A(x)=B R_{\alpha(x)}$, where $R_{\alpha}$ is the rotation of angle $\alpha$. Then the cocycle associated to any map $M \rightarrow G$ in a $C^{0}$ neighborhood of $A$ admits no invariant continuous subbundle, let alone a dominated splitting.

A simple proof goes as follows. Let $\xi_{0}: S^{1} \rightarrow \mathbb{P}^{1}$ be any continuous section in the real projective space $\mathbb{P}^{1}$, and $\xi_{1}: S^{1} \rightarrow \mathbb{P}^{1}$ be its push-forward:

$$
\xi_{1}(x)=A\left(f^{-1}(x)\right) \xi_{0}\left(f^{-1}(x)\right)=B R_{\alpha\left(f^{-1}(x)\right)} \xi_{0}\left(f^{-1}(x)\right) .
$$

Then $\operatorname{deg}\left(\xi_{1}\right)=2 \operatorname{deg}(\alpha)+\operatorname{deg}\left(\xi_{0}\right)$ (the factor 2 comes from the fact that we are considering maps to $\mathbb{P}^{1}$ instead of $S^{1}$ ). This implies that $\xi_{1} \neq \xi_{0}$, hence $\xi_{0}$ cannot be invariant. These topological arguments extend immediately to a $C^{0}$ neighborhood of $A$. 
In spite of this, non-zero Lyapunov exponents may occur in this setting. For example, if $f$ is an irrational rotation, $\alpha=\mathrm{id}$, and $\|B\|>1$ then, by Herman's subharmonicity argument ([7], see also [2]), the cocycle associated to $A$ has a positive exponent.

\section{Acknowledgements}

We lectured on these topics on several occasions. Recently, during the Workshop on Dynamical Systems held at the ICTP-Trieste in August 2001, we were able to improve our approach, resulting in substantially stronger statements. Questions asked by colleagues present at the ICTP, especially A. Avila, B. Fayad, K. Khanin, J. Mather, J. Milnor, C.G. Moreira, J. Palis, E. Pujals, M. Shishikura, Ya. Sinai, F. Takens, J.-C. Yoccoz, and L.-S. Young, motivated us to write this outline of the arguments, and helped improve the presentation.

\section{REFERENCES}

[1] Arnaud M.-C., The generic symplectic $C^{1}$ diffeomorphisms of 4-dimensional symplectic manifolds are hyperbolic, partially hyperbolic, or have a completely elliptic point, preprint Orsay, 2000.

[2] Avila A., Bochi J., A formula with some applications to the theory of Lyapunov exponents, www.preprint.impa.br, 2001.

[3] Bochi J., Genericity of zero Lyapunov exponents, www.preprint.impa.br, 2000.

[4] Bochi J., Viana M., A sharp dichotomy for conservative systems: zero Lyapunov exponents or projective hyperbolicity, in preparation.

[5] Bonatti C., Viana M., Lyapunov exponents with multiplicity 1 for deterministic products of matrices, www.preprint.impa.br, 2001.

[6] Bowen R., Equilibrium States and the Ergodic Theory of Anosov Diffeomorphisms, Lect. Notes in Math., Vol. 470, Springer Verlag, 1975.

[7] Herman M., Une méthode pour minorer les exposants de Lyapounov et quelques exemples montrant le caractère local d'un théorème d'Arnold et de Moser sur le tore de dimension 2, Comment. Math. Helvetici 58 (1983) 453-502.

[8] Ledrappier F., Quelques propriétés des exposants caractéristiques, École d'Été de Probabilités de Saint-Flour XII, 1982, Lect. Notes in Math., Vol. 1097, Springer Verlag, 1984.

[9] Mañé R., Oseledec's theorem from the generic viewpoint, in: Procs. Intern. Congress Math. Warszawa Vol. 2, 1983, pp. 1259-1276.

[10] Mañé R., The Lyapunov exponents of generic area preserving diffeomorphisms, in: Procs. Intern. Conf. Dynam. Syst., Montevideo, Pitman RNMS, Vol. 362, 1996, pp. 110-119.

[11] Oseledets V., A multiplicative ergodic theorem, Trudy Moscow Math. Obs. 19 (1968) 179210.

[12] Ruelle D., Analyticity properties of the characteristic exponents of random matrix products, Adv. Math. 32 (1979) 68-80. 